\title{
ESTIMATION OF ELEVATION DIFFERENCE BASED ON VEHICLE'S INERTIAL SENSORS
}

\author{
Jörg Barrho* Marcus Hiemer* Uwe Kiencke* \\ Takanori Matsunaga** \\ * Institute of Industrial Information Technology, \\ Universität Karlsruhe (TH), Germany \\ ** Mitsubishi Electric Corporation, Japan
}

\begin{abstract}
Modern navigation systems require reliable information about the vehicle position not only on plain. Roads possibly lying on top of each other, can be found in large cities. For this, it is necessary to know the transition from a road level to another one. GPS is not providing sufficiently accurate position information in these areas, because the received signals are disturbed. Therefore, inertial sensors can be employed. This paper presents a linear state space observer, which provides the road gradient. The covered height is obtained by multiplying the covered distance and the road gradient. Simulations and tests of the observer have shown, that reasonable results can be achieved. Copyright ${ }_{2005}$ IFAC
\end{abstract}

Keywords: Navigation systems, Inertial navigation, Road traffic, Gradients, Observers, Inertial sensors

\section{INTRODUCTION}

The measurement of the road gradient has to be carried out by means of special equipment, like an altimeter. This, however, is not a standard sensor of a modern middle class vehicle. Thus, an observer shall be developed, in order to estimate the road gradient by means of the standard sensor equipment of a modern vehicle, like acceleration signals and wheel speed sensors.

In order to determine the road gradient, a linear Luenberger-observer was developed. For that, a basic equation for road gradient estimation is presented. Afterwards, the observer design is shown. Next, an investigation of driving situations is carried out. Finally, results are presented and the necessity of verification is discussed.

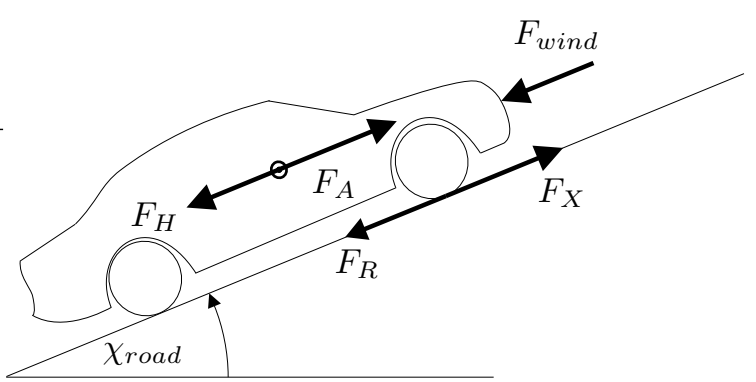

Fig. 1. Sketch of longitudinal forces of an ascending car

\section{MATHEMATICAL CONSIDERATION FOR ROAD GRADIENT ESTIMATION}

\subsection{Force Balance for Road Gradient Observation}

The effect of the lateral dynamics on road gradient estimation shall be neglected here. The vehicle body side slip angle is assumed to be zero. This assumption is true for straightforward on driving situations. 
Setting up the force balance of the forces displayed in Figure 1 yields the non-linear equation

$$
\begin{aligned}
\underbrace{m \cdot \dot{v}_{C o G}}_{F_{A}}= & F_{X}-\underbrace{m \cdot g \cdot \sin \chi_{R o a d}}_{F_{H}} \\
& -\underbrace{c_{W X r e s} \cdot v_{C o G}^{2}}_{F_{\text {wind }}}-F_{R}
\end{aligned}
$$

where $F_{R}$ is the rolling resistance force and can be approximated as follows (Mitschke and Wallentowitz, 2004)

$$
F_{R}=f_{R, 0}+f_{R, 1}\left(\frac{v_{C o G}}{30}\right)+f_{R, 4}\left(\frac{v_{C o G}}{30}\right)^{4}
$$

the coefficients $f_{R, 0}, f_{R, 1}$ and $f_{R, 4}$ are constant parameters depending on the employed tires.

\subsection{Linearization}

To reduce the computational complexity, a linear observer is employed here. Therefore, Eq. (1) has to be linearized. For the linearization, the following assumptions are made:

- the road gradient angle of public roads is limited to approx. $\pm 12^{\circ}$ (Halfmann, 2001), this yields $\sin \chi_{\text {Road }} \approx \chi_{\text {Road }}$

- the forces $F_{X}, F_{R}$ and $F_{\text {wind }}$ are merged into a resultant force $F_{\text {res }}=F_{X}-F_{\text {wind }}-F_{R}$. This is advantageous because the nonlinear terms $F_{\text {wind }}$ and $F_{R}$ become part of the input. The remaining state space model therefore is linear.

As a consequence of these assumptions Eq. (1) is simplified

$$
m \cdot \dot{v}_{C o G}=F_{\text {res }}-m \cdot g \cdot \chi_{\text {Road }} .
$$

\subsection{Linear State Space Equation}

Eq. (3) is now transformed to a state space model. The state vector $\underline{x}$ of the linear model contains the velocity $v_{C o G}$ and the road gradient angle $\chi_{\text {Road }}$. The input $\underline{u}$ is the resultant force $F_{\text {res }}$ :

$$
\underline{x}=\left[\begin{array}{c}
v_{C o G} \\
\chi_{\text {Road }}
\end{array}\right], \underline{u}=\left[\begin{array}{c}
F_{r e s} \\
0
\end{array}\right] .
$$

Then, the road gradient model can be written as

$$
\begin{aligned}
& \underbrace{\left[\begin{array}{c}
\dot{v}_{C o G} \\
\dot{\chi}_{S t r}
\end{array}\right]}_{\underline{\dot{x}}}= \\
& \underbrace{\left[\begin{array}{cc}
0 & -g \\
0 & 0
\end{array}\right]}_{\underline{A}} \underbrace{\left[\begin{array}{c}
v_{\text {CoG }} \\
\chi_{\text {Road }}
\end{array}\right]}_{\underline{x}}+\underbrace{\left[\begin{array}{cc}
\frac{1}{m} & 0 \\
0 & 0
\end{array}\right]}_{\underline{B}} \underbrace{\left[\begin{array}{c}
F_{\text {res }} \\
0
\end{array}\right]}_{\underline{u}},
\end{aligned}
$$

$$
\underbrace{\hat{v}_{C o G}}_{y}=\underbrace{\left[\begin{array}{c}
1 \\
0
\end{array}\right]^{T}}_{\underline{C}} \underbrace{\left[\begin{array}{c}
v_{\text {CoG }} \\
\chi_{\text {Road }}
\end{array}\right]}_{\underline{x}} .
$$

\section{ROAD GRADIENT OBSERVER DESIGN}

\subsection{Analysis of the system observability}

The observer design is carried out by means of the method of pole placement. This, however, requires an analysis of the system observability (Föllinger, 1994). The observability matrix $Q_{B}$ must have maximum rank. The matrix $\underline{Q}_{B}$ is calculated by means of Eq. (7), where $n$ denotes the system order.

$$
\underline{Q}_{B}=\left[\begin{array}{l}
\underline{C} \\
\underline{C} \underline{A} \\
\vdots \\
\underline{C} \underline{A}^{n-1}
\end{array}\right]
$$

For the system of Eqs. (5) and (6) $\underline{Q}_{B}$ becomes

$$
\underline{Q}_{B}=[\underline{\underline{C}} \underline{\underline{A}}]=\left[\begin{array}{rr}
1 & 0 \\
0 & -g
\end{array}\right] .
$$

As $\underline{Q}_{B}$ is square, the maximum rank can be checked by means of the determinant of $\underline{Q}_{B}$ :

$$
\begin{gathered}
\operatorname{det}\left(\underline{Q}_{B}\right) \stackrel{!}{\neq} 0 \\
\operatorname{det}\left(\underline{Q}_{B}\right)=\operatorname{det}\left[\begin{array}{rr}
1 & 0 \\
0 & -g
\end{array}\right]=-g \neq 0
\end{gathered}
$$

According to Eq. (10) the determinant of $Q_{B}$ is $-g$ which is earth's gravitational acceleration. Therefore, the rows of matrix $\underline{Q}_{B}$ are linearly independent and the linear road gradient model, see Eq. (5), is observable. Thus, the observer design by means of pole placement is feasible.

\subsection{Observer design and structure}

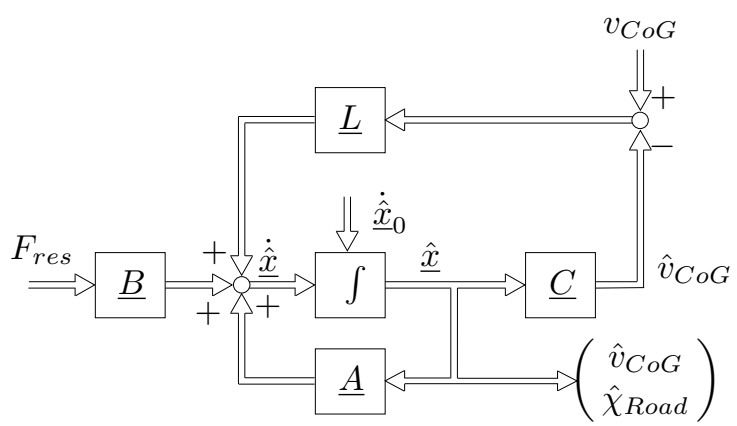

Fig. 2. Structure of the developed LuEnBERGERobserver 
Since the system order is $n=2$, the Luenbergerobserver matrix $\underline{L}$ consists of two elements $l_{1}$ and $l_{2}$. In order to calculate the elements of matrix $\underline{L}$, the poles of the observed system must be placed appropriately. Its characteristic polynomial is

$$
\begin{aligned}
& \operatorname{det}(s \underline{I}-\underline{A}+\underline{L C})= \\
&=\operatorname{det}\left(\left[\begin{array}{ll}
s & 0 \\
0 & s
\end{array}\right]-\left[\begin{array}{rr}
0 & -g \\
0 & 0
\end{array}\right]+\left[\begin{array}{ll}
l_{1} & 0 \\
l_{2} & 0
\end{array}\right]\right) \\
&=\operatorname{det}\left(\begin{array}{cc}
s+l_{1} & g \\
l_{2} & s
\end{array}\right) \\
&=s^{2}+s \cdot l_{1}-g \cdot l_{2}
\end{aligned}
$$

The eigenvalues are denoted as $\lambda_{1}$ and $\lambda_{2}$ and are chosen according to the following equation

$$
\begin{aligned}
s^{2}+s \cdot l_{1}-g \cdot l_{2} \stackrel{!}{=}\left(s-\lambda_{1}\right)\left(s-\lambda_{2}\right) \\
=s^{2}-s \cdot\left(\lambda_{1}+\lambda_{2}\right)+\lambda_{1} \cdot \lambda_{2}(13)
\end{aligned}
$$

For pole-placement, the coefficients of Eq. (13) are compared. This yields the elements of the observer-matrix $\underline{L}$, see Eq. (14).

$$
l_{1}=-\lambda_{1}-\lambda_{2}, \quad l_{2}=\frac{-\lambda_{1} \cdot \lambda_{2}}{g}
$$

Next, the eigenvalues $\lambda_{1}$ and $\lambda_{2}$ are determined. For this, a simulation model with the structure shown in Figure 2 was implemented. The following strategy for pole placement was employed to achieve suitable values of $\lambda_{1}$ and $\lambda_{2}$ :

- the eigenvalues must be negative, otherwise the observer system becomes unstable.

- if the eigenvalues are too far left in the s-plane, the observer becomes sensitive to noise.

- if the eigenvalues are too close to the imaginary axis, the observer becomes too slow. Then, it would not be able to follow the driving state of the vehicle properly (e.g. uphill and downhill driving).

Considering these constraints, and running a variety of simulations, the eigenvalues were fixed to

$$
\begin{aligned}
& \lambda_{1}=-2 \\
& \lambda_{2}=-3 .
\end{aligned}
$$

\section{DETERMINATION OF DRIVING SITUATION}

Here, driving situation means the driving state, like uphill and downhill driving, accelerating or braking. Distinguishing these driving states is essential, in order to obtain proper road gradient results. Since the above described road gradient estimation calculates a "road gradient" even when the vehicle is braking or accelerating on plane road, an assessment of the resulting road gradient has to be developed.

The "wrong road gradient" is obtained due to the vehicle dynamics. Especially, due to differences of $a_{X}$ (vehicle longitudinal acceleration) and acceleration of the averaged wheel speeds. This difference is caused by wheel slip. On the other hand, the vehicle is also pitching during acceleration and braking maneuvers. So, the road gradient angle can be fudged by the pitch angle, which occurs during braking or acceleration due to wheel load changes (Daiß, 1996).

In order to detect the driving state, the following information are assessed:

- $a_{X}$ signal, provided by the acceleration sensor in vehicle's longitudinal direction

- averaged wheel speed acceleration $\dot{\bar{\omega}}_{R}$ is the time-derivated signal of the averaged value of the four wheel speeds $\bar{\omega}_{R}$

- pitch angle $\chi$, which is estimated by an appropriated model, (Daiß, 1996).

In (Kiencke and Nielsen, 2000) the difference of $a_{X}$ and $\dot{\bar{\omega}}_{R}$ is utilized for estimation of the road gradient angle. This approach, however, requires i. a. two low-pass filters, in order to obtain a result. This method will not be discussed further. Nevertheless, the difference shown in Equation (16), shall be used for driving state evaluation.

$$
\operatorname{diff}(n)=a_{X}(n)-\dot{\bar{\omega}}_{R}(n)
$$

The evaluation of a variety of test runs conducted with a test vehicle provides specific mean values of diff $(n)$ for uphill and downhill driving. These values are listed in Table (1).

$$
\begin{gathered}
\frac{\text { value of } \text { diff }_{\text {mean }}}{\geq 0.9} \text { driving situation } \\
\leq-0.2 \quad \text { uphill driving } \\
\text { Table 1. Evaluation result of mean value } \\
\text { of } \operatorname{diff}(n)
\end{gathered}
$$

In order to gain an algorithm, which is capable to distinguish driving states continuously, only the last 200 measurement values of $a_{X}$ and $\dot{\bar{\omega}}_{R}$ are employed. This represents a time span of 2 seconds. Using less than 200 measurements causes a fluctuating result. On the other hand, "old" values (if much more than 200 measurements are used) influence the value diff $_{\text {mean }}$. Then, no time-variant changes can be detected. Therefore, diff $_{\text {mean }}$ is either too big or too small. A proper differentiation of driving situation becomes critical. Thus, a moving average calculation of diff $(n)$ is carried out (Eq. (17)).

$$
\operatorname{diff}_{\text {mean }}(n)=\frac{\sum_{i=n-200}^{n} \operatorname{diff}(i)}{200}
$$




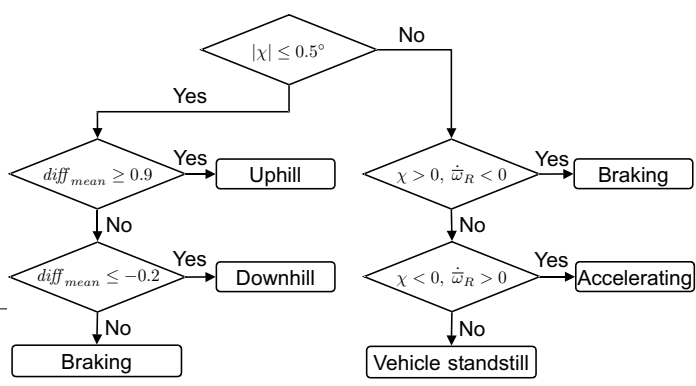

Fig. 3. Flow chart of the algorithm for distinguishing driving states

Furthermore, the evaluation of the test runs yields a pitch angle limit for differing between uphill / downhill driving and accelerating / braking. Within the limits of $-0.5^{\circ} \leq \chi \leq 0.5^{\circ}$ the vehicle is moving up-/downhill. Otherwise, the vehicle is accelerating or braking. Therefore, a stationary vehicle motion was assumed. Due to this classification of driving states and the assumption of stationary motion, the developed algorithm is not capable to recognize e.g. acceleration during uphill driving.

In order to distinguish braking and accelerating, the definitions shown in Table (2) are utilized.

$$
\begin{aligned}
& \begin{array}{ccc}
\text { pitch angle } \chi & \dot{\bar{\omega}}_{R} & \text { driving situtation } \\
\hline>0 & <0 & \text { braking }
\end{array} \\
& \begin{array}{cccc} 
& <0 & >0 & \text { accelerating } \\
\hline \text { Table } & 2 . & \text { Conditions of brak- }
\end{array} \\
& \text { ing/accelerating }
\end{aligned}
$$

The described facts and information are combined. So, the flow chart in Figure (3) is obtained. This flow chart shows the coherences to distinguish different driving states.

Furthermore, the termination block braking on the left side in Figure (3) results from test runs. The developed system to distinguish driving states follows the vehicle dynamics sufficiently, even if the vehicle is moving uphill or downhill and braking at the same time. However, the system requires a settling time of approx. 5-10 s.

\section{CALCULATION OF ELEVATION DIFFERENCE}

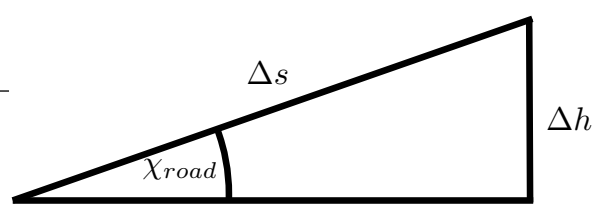

Fig. 4. Calculation of the climbed hight

As a result of the last section, the elevation change can be calculated, if the algorithm detects uphill or downhill motion of the vehicle. In figure (4) the

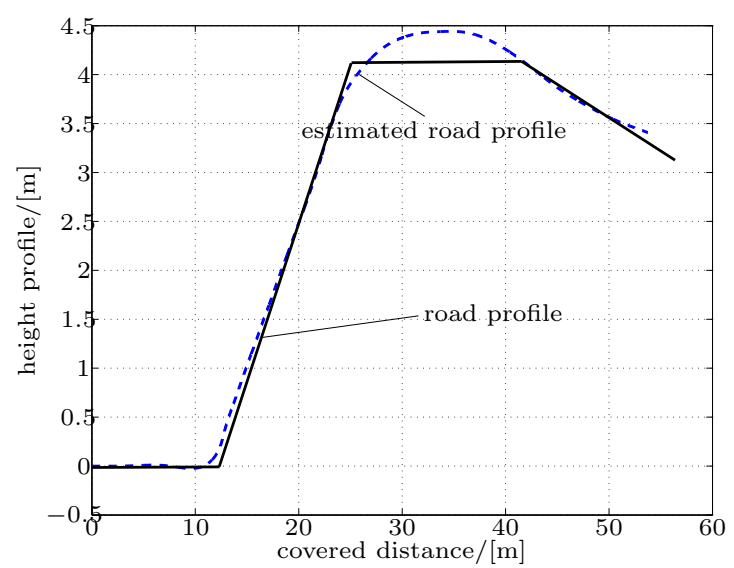

Fig. 5. Road gradient of a test course with a defined gradient of $33 \%$

coherences are displayed. The elevation change $\Delta h$ is calculated by means of

$$
\Delta h \approx \Delta s \cdot \chi_{\text {road }}
$$

assuming the road gradient is below $12^{\circ}$. The road gradient $\chi_{\text {road }}$ is obtained by the Luenbergerobserver. $\Delta s$ denotes the covered distance and is determined by

$$
\Delta s=\bar{\omega}_{R} \cdot r_{\text {stat }} \cdot T,
$$

where $\bar{\omega}_{R}$ is the averaged wheel speed, $r_{\text {stat }}$ denotes the static wheel radius and $T$ is the sample time. Therefore, the height profile can be estimated. A simulation result is presented in the next subsection.

\section{RESULTS}

The height profile of a road calculated on basis of the estimated road gradient is displayed in Figure (5). The test drive was carried out on a test course with a defined road gradient. Starting on a flat road, after $t=2.5 \mathrm{~s}$ the car enters an inclined plane with a gradient of $33 \%$ ( $\chi_{\text {road }} \approx$ $\left.18^{\circ}\right)$. The car moves on this inclined plane for approximately $10 \mathrm{~s}$, returns to a flat road again and moves downhill at the end. The estimated road gradient of the first ramp is approximately $30 \%$. The deviation to the real road gradient is caused by the linear approximation which is not valid any more. At the end of the inclined plane the vehicle suddenly returns to a flat road. The pitch angle during this transition is responsible for the deviation of the maximum height $(\approx 10 \%)$. All in all, even for large road gradients, the linear observer provides very good results.

In Figure (6) a simulation result of a road gradient angle estimation is displayed. The input values of the simulation are provided from a real test run. It 

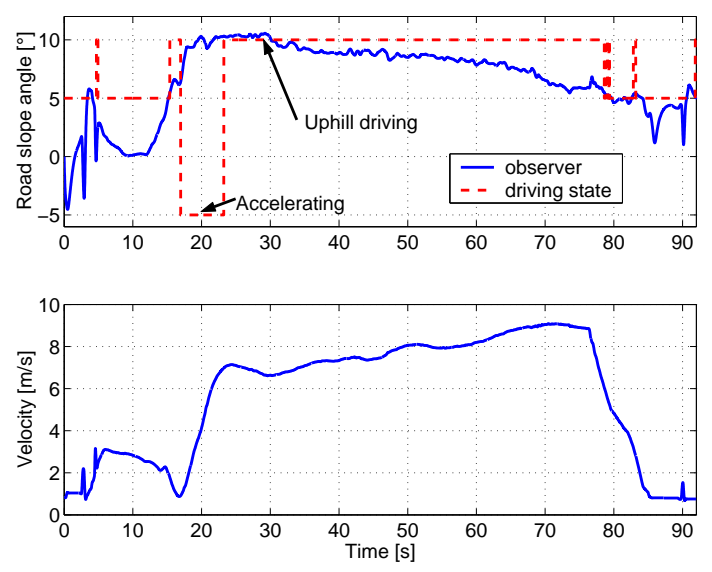

Fig. 6. Simulation result of road gradient angle estimation

was conducted on a road with an approx. gradient angle of $8-11^{\circ}$.

In the upper diagram of Figure (6) the estimated road gradient angle is shown. Note that the longitudinal wheel force (one of the input values of the simulation) is estimated as $F_{X}=0.9 \cdot m \cdot a_{X}$. The dashed line represents the identified driving status. In the beginning, the result of driving status identification is not reliable, because not enough values for diff mean -calculation are available. Furthermore, the above mentioned settling time can be seen, if the upper and the lower diagrams are compared. The lower diagram shows the velocity $\bar{\omega}_{R}$ of the vehicle. The upper diagram, however, shows that the road gradient angle estimation is in the range between $8-11^{\circ}$, thus the observer approach works correctly and the algorithm distinguishes different driving situations properly.

\section{CONCLUSION}

A model based observer design for road gradient estimation is presented. Therefore, a linearized equation of the longitudinal vehicle forces is utilized. Based on this equation, the observer design is carried out according to the Luenberger-approach. Simulations of the designed observer have shown, that reasonable results can be achieved leading to an improved positioning for navigation systems. However, further tests have to be conducted to verify the reliability of the presented methodology.

In oder to distinguish different driving situations, an algorithm is shown which can detect these situations. Knowing these driving situations might provide a basis for situation-based parameter estimation such as the vehicle mass (Halfmann, 2001).

\section{REFERENCES}

Daiß, A. (1996). Beobachtung fahrdynamischer Zustände und Verbesserung einer ABS- und Fahrdynamikregelung. VDIFortschrittsberichte, Reihe 12, Nr. 283. VDIVerlag. Dsseldorf.

Föllinger, O. (1994). Regelungstechnik. 8. ed.. Hüthig Verlag. Heidelberg.

Halfmann, C. (2001). Adaptive semiphysikalische Echtzeitsimulation der Kraftfahrzeugdynamik im bewegten Fahrzeug. VDI-Fortschrittsberichte, Reihe 12, Nr. 467. VDI-Verlag. Dsseldorf.

Kiencke, U. and L. Nielsen (2000). Automotive Control Systems. Springer-Verlag. Berlin, Heidelberg, New York.

Mitschke, M. and H. Wallentowitz (2004). Dynamik der Kraftfahrzeuge. 4. ed.. Springer Verlag. Berlin. 